\title{
Intravenous Immunoglobulin in the Management of Lupus Erythematosus Panniculitis
}

\author{
Nada G. AlQadri ${ }^{1}$, Bayan AlNooh ${ }^{2}$, Malak M. AlTewerki ${ }^{3}$, Ahmad Almotairi ${ }^{4}$, Saad Alajlan ${ }^{5}$ \\ 1. Department of Dermatology, Alfaisal University College of Medicine, Riyadh, SAU 2. Department of Dermatology, \\ King Khalid University Hospital, Riyadh, SAU 3. Department of Neuroscience, King Faisal Specialist Hospital and \\ Research Centre, Riyadh, SAU 4. Department of Pathology, King Saud University Medical City, Riyadh, SAU 5. \\ Department of Dermatology, King Faisal Specialist Hospital and Research Centre, Riyadh, SAU
}

Corresponding author: Saad Alajlan, salajlan@kfshrc.edu.sa

\begin{abstract}
Lupus erythematosus panniculitis (LEP) is a rare variant of cutaneous lupus erythematosus (CLE). It is characterized by the presence of a chronic inflammatory process involving the deep dermis and subcutaneous tissues. It commonly presents as deep indurated nodules or sharply demarcated plaques. Antimalarial medications are considered first-line therapy for most cases of LEP while systemic corticosteroids are saved for more resistant lesions. Intravenous immunoglobulin (IVIG) is made up of concentrated polyclonal immunoglobulin G (IgG) fractionated from the blood of healthy blood donors. Nowadays, it is used for the treatment of numerous autoimmune and systemic inflammatory diseases. In this case, we report the case of a female with multiple LEP and discoid lupus erythematosus (DLE) lesions
\end{abstract} refractory to multiple standard therapy modalities that responded dramatically to IVIG.

Received 01/14/2020 Review began 01/21/2020 Review ended 01/22/2020 Published 01/27/2020

(c) Copyright 2020 AlQadri et al. This is an open access article distributed under the terms of the Creative Commons Attribution License CC-BY 3.0., which permits unrestricted use, distribution, and reproduction in any medium, provided the original author and source are credited.
Categories: Dermatology, Internal Medicine, Rheumatology

Keywords: lupus, lupus erythematosus panniculitis, lupus erythematosus profundus, cutaneous lupus, sle, intravenous immunoglobulin, ivig, chronic cutaneous lupus

\section{Introduction}

Lupus erythematosus panniculitis (LEP), also known as lupus profundus (LP), is a rare subtype of lupus erythematosus (LE) consisting of $1 \%-3 \%$ of patients with cutaneous lupus erythematosus (CLE) [1]. LEP is a chronic inflammatory process that mainly involves the deep dermis and subcutaneous tissues, usually presenting as deep indurated nodules or sharply demarcated plaques [2]. It can either present as the sole manifestation of the disease or in association with discoid lupus erythematosus (DLE) or systemic lupus erythematosus (SLE). The most commonly involved areas include the lateral aspects of the arms and shoulders, buttocks, trunk, breast, face, and scalp [3]. Antimalarial medications are considered first-line therapy for most cases of LEP; meanwhile, systemic corticosteroids are saved for resistant lesions [2,4]. Intravenous immunoglobulin (IVIG) is derived from the blood of healthy blood donors and is made up of concentrated polyclonal immunoglobulin G (IgG). It was initially used as a treatment for patients with immunodeficiency. Nowadays, it is used as an off-label therapy for a wide variety of autoimmune and systemic inflammatory diseases, especially in dermatology. However, its mechanism of action is unknown [5-6]. Herein, we present a case of a female with multiple refractory LEP and DLE lesions over the face and scalp, respectively, which responded dramatically to IVIG.

\section{Case Presentation}

In September 2016, a 34-year-old Saudi female was referred to the department of dermatology at King Faisal Specialist Hospital and Research Center (KFSHRC) with the aim of establishing a suspected diagnosis of LEP and for further management. The patient had been following up at multiple hospitals and has tried different treatments, including intralesional and systemic corticosteroids, hydroxychloroquine (HCQ), methotrexate, and mycophenolate mofetil with no satisfactory control over the lesions. On presentation, the main complaint of the patient was the presence of painful skin lesions over the face and two painful localized areas of alopecia over the scalp for more than 10 years. On physical examination, the patient had multiple, indurated, erythematous, tender plaques on the cheeks and one lesion on the forehead, measuring $0.5 \mathrm{~cm}$ in size. On her scalp, over the vertex, there were two well-defined erythematous, indurated, scarring patches of alopecia measuring $1.5 \times 3 \mathrm{~cm}$ and $1 \mathrm{~cm}$ in size, with no other involved areas. Laboratory investigations conducted at the hospital revealed leucopenia, $3.89 \times 109 / \mathrm{L}$, and positive antinuclear antibodies (ANA) (titer 1:640 with a speckled pattern). However, anti-ds-DNA, anti-SSA (Ro), anti-Smith, anti-SCL-70, anti-JO1, and anti-RNP antibodies were negative. A skin biopsy taken from the cheek revealed lobular panniculitis with lymphocytic, including plasma cell infiltrates, dermal perivascular and periadnexal lymphocytic infiltrates with mucin deposition, follicular plugging, vacuolar-type interface dermatitis, and epidermal atrophy (Figure 1 and Figure 2). 


\section{Cureus}

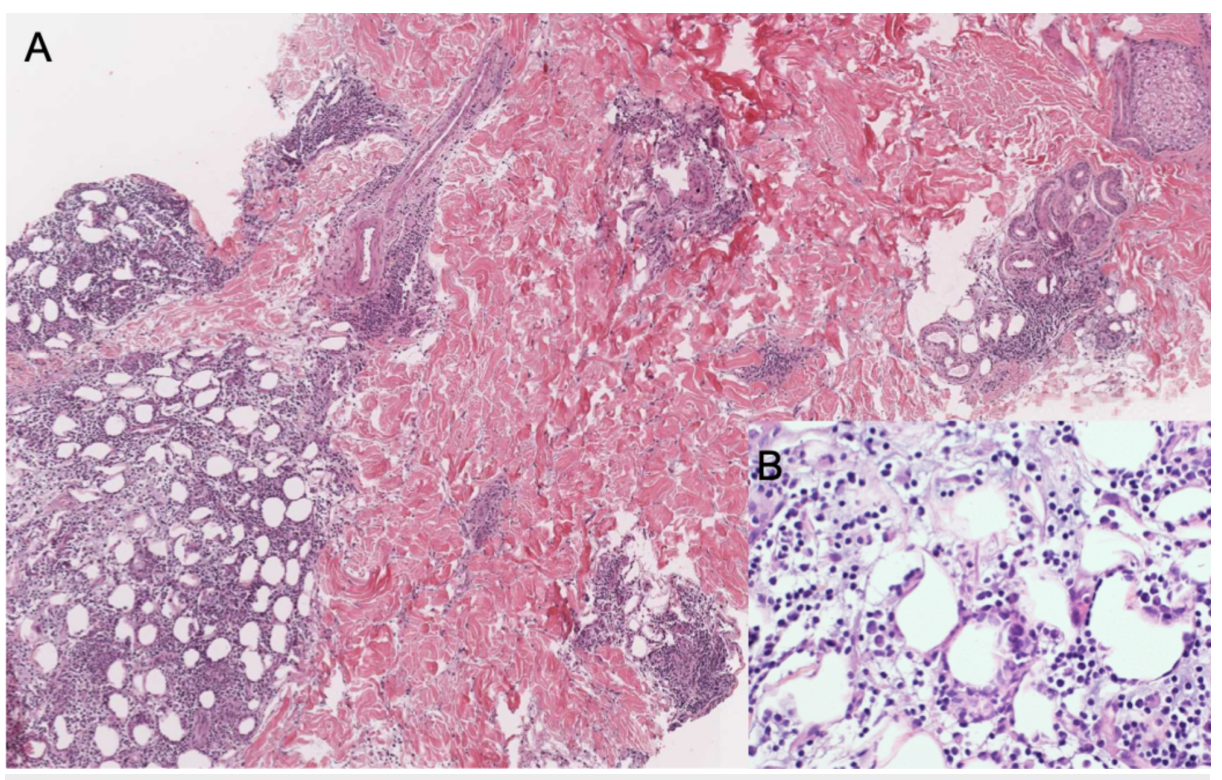

\section{FIGURE 1: Thirty-six-year-old lady; Skin punch biopsy from the right}

cheek

A: Section from subcutaneous tissue shows lobular panniculitis and perivascular and periadnexal lymphocytic infiltration. B: The infiltrate is predominantly lymphocytic with scattered plasma cells

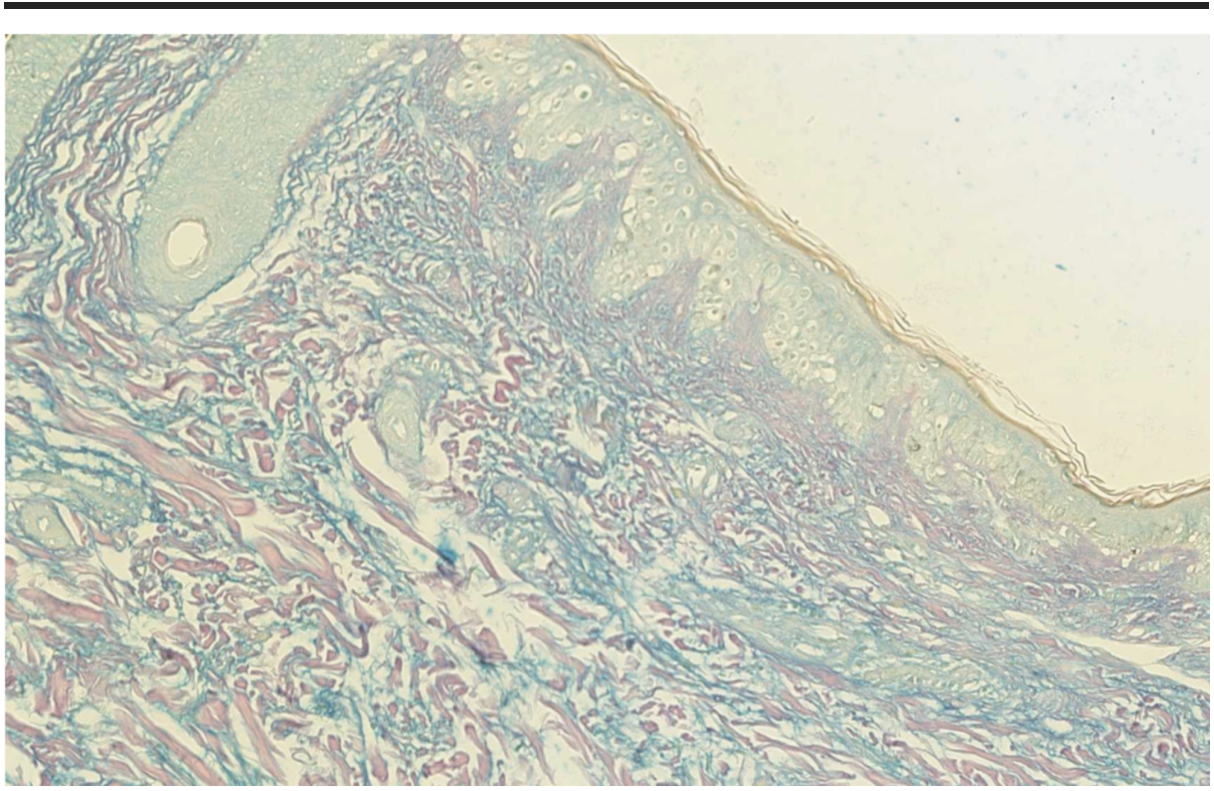

\section{FIGURE 2: Thirty-six-year-old lady; Skin punch biopsy from the right cheek showing increased dermal mucin}

A biopsy from the scalp lesion revealed prominent fat necrosis with a membranocystic pattern, dermal periadnexal and perivascular lymphocytic infiltrate, follicular plugging, vacuolar-type interface dermatitis, and epidermal atrophy. The biopsy findings were consistent with the diagnosis of LEP over the cheeks and forehead and DLE over the scalp. During her follow-up, the patient was referred to the rheumatology clinic where she was cleared of SLE. Treatment was initiated with systemic corticosteroids at $30 \mathrm{mg} / \mathrm{day}$, which was tapered down by $5 \mathrm{mg}$ every three days, and intralesional corticosteroids $10 \mathrm{mg} / \mathrm{ml}$ for DLE lesions over the scalp. One month later, both LEP and DLE lesions were still painful and erythematous. Therefore, HCQ 400 $\mathrm{mg} /$ day was added and the systemic corticosteroids dose was increased to $40 \mathrm{mg} /$ day, which was tapered down to $20 \mathrm{mg} /$ day, and she was given intralesional corticosteroids for DLE lesions (10 mg/ml). Two months later, she was still complaining of pain and itching over the scalp lesions and pain over the facial lesions. The systemic corticosteroid dose was increased to $40 \mathrm{mg} / \mathrm{day}$, which was tapered down to $30 \mathrm{mg} /$ day. Intralesional corticosteroids and HCQ were continued. The patient was seen one month later, however, LEP 


\section{Cureus}

lesions have become more painful and there was little improvement in the pain over the DLE lesions, so the systemic corticosteroids were increased to $50 \mathrm{mg} /$ day for one week, followed by $45 \mathrm{mg} /$ day for the next week, and $40 \mathrm{mg} /$ day to be continued for two weeks. Also, the intralesional corticosteroids for DLE lesions were increased to $20 \mathrm{mg} / \mathrm{ml}$ and HCQ was continued. One month later, there was no improvement, thus, rituximab infusions at a rate of 1 gram every two weeks for a total dose of 2 grams were initiated. Systemic corticosteroids $40 \mathrm{mg} /$ day, intralesional corticosteroids, and HCQ were continued. The patient was seen 15 weeks later, at which she had HCQ for seven months, systemic corticosteroids for nine months, more than four sessions of intralesional corticosteroids, and two rituximab infusions, the last being one month prior to her appointment; there was no improvement. Thus, cyclosporine 350-mg/day was started and monthly intralesional corticosteroids for DLE lesions and HCQ were continued. Two months later, she reported that she was unable to tolerate cyclosporine and discontinued its use after 20 days and that her facial lesions have grown larger. Therefore, IVIG monthly cycles were started three months after the last rituximab infusion at $2 \mathrm{mg} / \mathrm{kg}$ infused over three days per month along with topical and intralesional corticosteroids for DLE lesions and HCQ. After four cycles of IVIG, LEP lesions had resolved with residual

hyperpigmentation and atrophy (Figure 3) and the DLE lesions over the scalp had some new hair regrowth (Figure 4). IVIG, topical and intralesional corticosteroids for DLE lesions, and HCQ were continued. After 12 cycles of IVIG, LEP lesions have remained in remission and no new lesions have developed. However, she was still complaining of pain and itching over the scalp lesions, which occur one week after receiving the intralesional corticosteroid injections, therefore, mycophenolate mofetil $1000 \mathrm{mg} /$ day was added. It was later increased to $2000 \mathrm{mg}$ /day due to the same complaint; also, intralesional corticosteroids were increased from $20 \mathrm{mg} / \mathrm{ml}$ to $40 \mathrm{mg} / \mathrm{ml}$. IVIG tapering off will be initiated by increasing the intervals between single cycles by two weeks each time until a 16-week interval is reached where the drug will be discontinued; Moreover, HCQ, mycophenolate mofetil, and topical and intralesional corticosteroids for DLE lesions will be continued. Also, the patient was referred to plastic surgery for an auto-fat transplant.

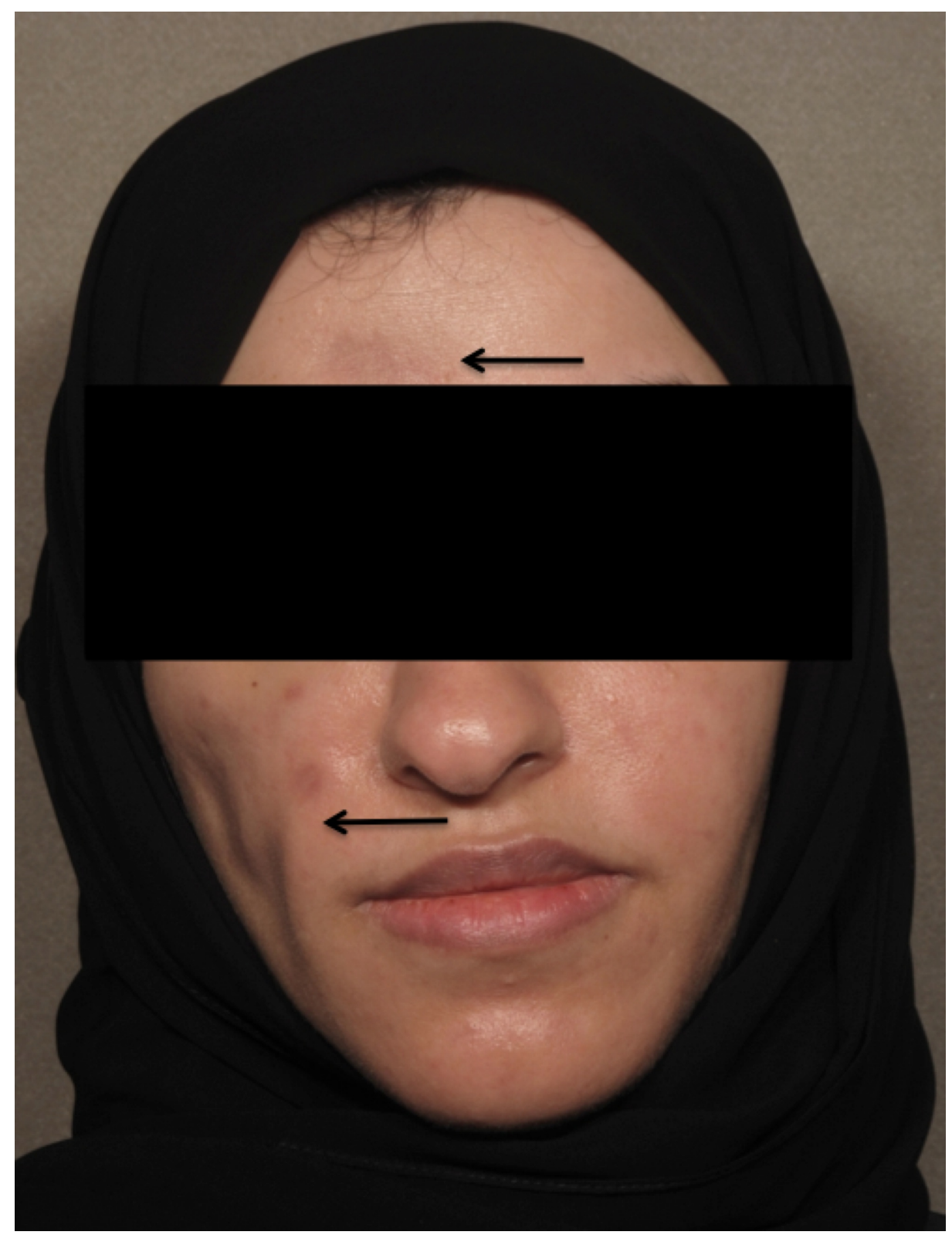




\section{Patient Timeline}

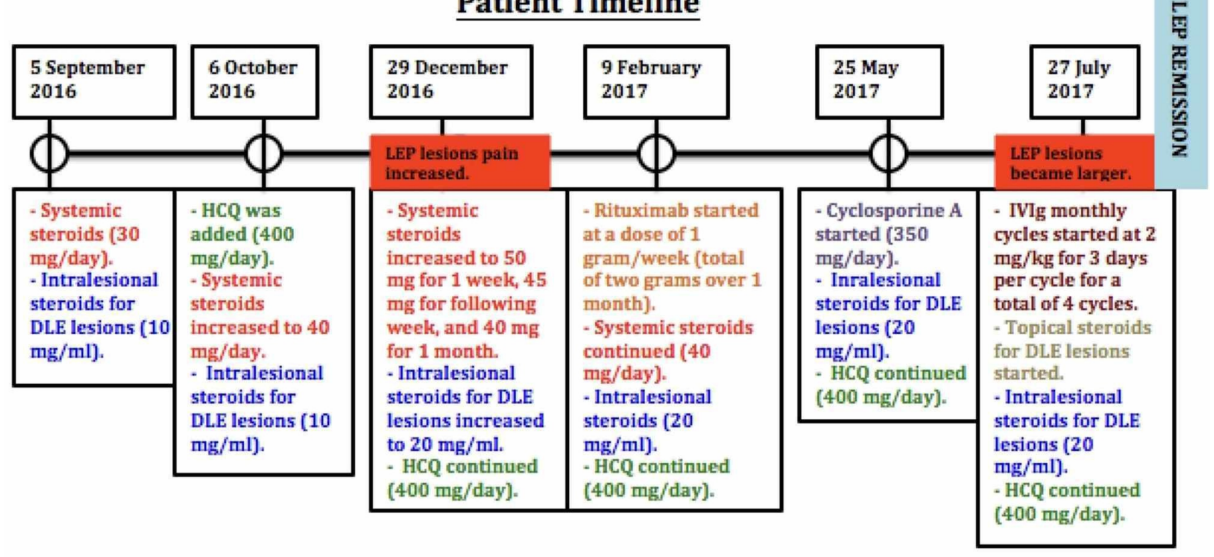

FIGURE 4: Patient treatment timeline

\section{Discussion}

LEP is a rare subtype of chronic CLE [7]. It was first described by Kaposi in 1883 and was later named "lupus erythematosus panniculitis" by Irgang in 1940 [8]. Although the terms LEP and lupus profundus are used interchangeably, it is suggested that only when the subcutaneous inflammatory process is accompanied by overlying DLE lesions is it called lupus profundus [7]. LEP most commonly affects women between the ages of 30 and 60 years. It is characterized by the presence of deep, tender subcutaneous nodules or plaques located in adipose-rich areas such as the arms and buttocks. Histopathologically, it is characterized by the presence of lymphocytic infiltrates and hyaline necrosis of the fat lobules [7-9]. The gold standard for establishing a diagnosis is by a histopathological examination of a lesional skin specimen. LEP has a relapsing course and new lesions may develop in previously affected areas or in new areas [10]. The healing of the lesions leaves behind irreversible lipoatrophy, which can be a major source of disfigurement and depression [11]. Commonly utilized treatments include antimalarial drugs, such as chloroquine or HCQ for mild cases of separate LEP. Topical and intralesional corticosteroids are also used. In refractory cases, methotrexate, cyclosporine A, cyclophosphamide, thalidomide, and the monoclonal antibody rituximab have been used [10-11]. IVIG is a fractionated blood product consisting of pooled IgG antibodies. Over 10,000 healthy blood donors are required for the production of a single batch. Initially, it was used for the treatment of primary and secondary immune deficiencies; however, its use has expanded tremendously in the past few decades. Effects produced by IVIG include the blockage and degradation of complement, induction of immunomodulatory Fc receptors, inhibition of B-cells, altering T-cell function, migration, and cytokine production. It is either used as a monotherapy or with an immunosuppressant [6]. There are multiple cases in the literature reporting the successful treatment of refractory DLE lesions using IVIG [6]. However, there is only one report in the literature reporting the successful treatment of LEP using IVIG [11]. The high cost of IVIG is a major drawback to its use [5]. The common side effects associated with IVIG administration include flu-like symptoms, such as flushing, nausea, fever, malaise, and lethargy; however, these side effects can be overcome easily by slowing down the infusion rate and by the administration of antihistamines and non-steroidal anti-inflammatory drugs (NSAIDs) [12]. In this case, the disease follows a chronic course and is resistant to multiple widely used treatments. Systemic corticosteroids, antimalarials, methotrexate, rituximab, cyclosporine A, and mycophenolate mofetil resulted in limited success. IVIG resulted in complete resolution of LEP lesions; however, DLE lesions required more aggressive immunosuppression using mycophenolate mofetil, which was added later.

\section{Conclusions}

LEP is a rare variant of CLE. Antimalarial drugs, systemic corticosteroids, and immunosuppressive medications are often effective in the treatment of LEP. In this case, IVIG has proven effective when other modalities provided suboptimal control. Therefore, IVIG can be used when other standard therapy modalities have failed to control the disease. However, large clinical trials are needed to prove the efficacy of IVIG in the treatment of LEP. 


\section{Additional Information}

Disclosures

Human subjects: Consent was obtained by all participants in this study. King Faisal Specialist Hospital and Research Center issued approval 2180267. Your application to submit manuscript /abstract/ poster/oral presentation titled "Intravenous Immunoglobulin in the Management of Lupus Erythematosus Panniculitis: A Case Report" was received by the Office of Research Affairs (ORA) on 15 August 2018. It has been assigned Publication No. 2180267 (No. appears in the upper left side of the publication submission form) and is cleared for publication (copy attached). Please use this number in your future correspondences with ORA regarding this publication. Please update ORA when this publication is approved or submitted to another meeting. Conflicts of interest: In compliance with the ICMJE uniform disclosure form, all authors declare the following: Payment/services info: All authors have declared that no financial support was received from any organization for the submitted work. Financial relationships: All authors have declared that they have no financial relationships at present or within the previous three years with any organizations that might have an interest in the submitted work. Other relationships: All authors have declared that there are no other relationships or activities that could appear to have influenced the submitted work.

\section{References}

1. Park HS, Choi JW, Kim BK, Cho KH: Lupus erythematosus panniculitis: clinicopathological, immunophenotypic, and molecular studies. Am J Dermatopathol. 2010, 32:24-30. 10.1097/DAD.0b013e3181b4a5ec

2. Gondane S, Kothiwala R, Dangi S, Meherda A: Lupus erythematosus panniculitis in pregnancy. Indian J Dermatol. 2015, 60:637. 10.4103/0019-5154.169164

3. Fraga J, García-Díez A: Lupus erythematosus panniculitis. Dermatol Clin. 2008, 26:453-463. 10.1016/j.det.2008.06.002

4. Braunstein I, Werth VP: Update on management of connective tissue panniculitides . Dermatol Ther. 2012, 25:173-182. 10.1111/j.1529-8019.2012.01489.x

5. Prabagar MG, Choi HJ, Park JY, Loh S, Kang YS: Intravenous immunoglobulin-mediated immunosuppression and the development of an IVIG substitute. Clin Exp Med. 2014, 14:361-373. 10.1007/s10238-013-0255-4

6. Ky C, Swasdibutra B, Khademi S, Desai S, Laquer V, Grando SA: Efficacy of intravenous immunoglobulin monotherapy in patients with cutaneous lupus erythematosus: results of proof-of-concept study. Dermatol Reports. 2015, 16:5804. 10.4081/dr.2015.5804

7. Jaczynska K, Wonziniacka A, Waszczykowska E, Zebrowska A: Lupus erythematosus panniculitis resistant to standard treatment, complicated with macrophage activation syndrome. Postepy Dermatol Alergol. 2017, 34:281-283. 10.5114/ada.2017.67852

8. Zhang R, Dang X, Shuai L, He Q, He X, Yi Z: Lupus erythematosus panniculitis in a 10-year-old female child with severe systemic lupus erythematosus. Medicine (Baltimore). 2018, 97:e9571. 10.1097/MD.0000000000009571

9. Guissa VR, Trudes G, Jesus AA, Aikawa NE, Romiti R, Silva CA: Lupus erythematosus panniculitis in children and adolescents. Acta Rheumatol Port. 2012, 37:82-85.

10. Bednarek A, Bartoszak L, Samborski W: Case report on a patient with lupus panniculitis [Article in Polish] . Postepy Dermatol Alergol. 2015, 32:59-62. 10.5114/pdia.2014.40958

11. Espírito Santo J, Gomes MF, Gomes MJ, et al.: Intravenous immunoglobulin in lupus panniculitis . Clin Rev Allergy Immunol. 2010, 38:307-318. 10.1007/s12016-009-8162-X

12. Guo Y, Tian X, Wang X, Xiao Z: Adverse effects of immunoglobulin therapy . Front Immunol. 2018, 8:1299. 10.3389/fimmu.2018.01299 